SECTION 2. Applied mathematics. Mathematical modeling.

Alexander Danilovich Chernyshov

Doctor of physico-mathematical Sciences, professor The Voronezh State University of Engineering Technology, Russia. chernyshovad@mail.ru

Vitalij Valerevich Gorjajnov Candidate of physico-mathematical Sciences, associate prof. The Voronezh State University of Architecture and Civil Engineering, Russia. gorvit77@mail.ru

Oleg Alexandrovich Chernyshov Assistant The Voronezh State University of Engineering Technology, Russia. chernyshovad@mail.ru

\title{
CALCULATION OF FLIGHT OF A SPACECRAFT ON THE EXOATMOSPHERIC PORTION OF THE TRAJECTORY BY THE METHOD RAPID EXPANSIONS
}

Abstract: The problem of the motion of a spacecraft on the exoatmospheric portion of the trajectory. An analytical solution by rapid expansions. Shows the absolute error of the trajectory of a spacecraft, its speed and acceleration, which showed high efficiency of the method rapid expansions.

Keywords: Spacecraft, flight trajectory, rapid expansion, an analytical solution.

\section{УДК 629.78}

\section{РАСЧЕТ ПОЛЕТА КОСМИЧЕСКОГО КОРАБЛЯ НА ВНЕАТМОСФЕРНОМ УЧАСТКЕ ТРАЕКТОРИИ МЕТОДОМ БЫСТРЫХ РАЗЛОЖЕНИЙ}

Аннотация: Рассмотрена задача о движении космического корабля на внеатмосферном участке траектории. Предложено аналитическое решение методом быстрых разложений. Приведены абсолютные погрешности траектории космического корабля, его скорости и ускорения, которые показали высокую эффективность метода быстрых разложений.

Ключевые слова: космический корабль, траектория полета, быстрые разложения, аналитическое решение.

При расчетах траектории полетов космических кораблей обычно используют конечно-разностные методы $[1,2]$. В данной работе предлагается новый аналитический метод - метод быстрых разложений [3], позволяющий с высокой точностью при минимальных затратах на ЭВМ определить решение в аналитическом виде [4-10].

Уравнения движения корабля запишем в декартовой системе координат с началом в центре Земли:

$$
\ddot{x}+\frac{\alpha x}{\left(x^{2}+y^{2}+z^{2}\right)^{3 / 2}}=P_{x}, \ddot{y}+\frac{\alpha y}{\left(x^{2}+y^{2}+z^{2}\right)^{3 / 2}}=P_{y}, \ddot{z}+\frac{\alpha z}{\left(x^{2}+y^{2}+z^{2}\right)^{3 / 2}}=P_{z},
$$

где $\alpha=g R_{c}^{2} ; R_{c}$-радиус Земли; $g$-ускорение свободного падения; $\left(P_{x}, P_{y}, P_{z}\right)$-вектор условно действующей силы, отнесенной к массе корабля: 


$$
\begin{gathered}
P_{x}=-a \omega^{2} \cos \omega t+\frac{\alpha a \cos \omega t}{\left((a \cos \omega t)^{2}+(a \sin \omega t)^{2}+\left(R_{c}+h+w^{*} t\right)^{2}\right)^{(3 / 2)}}, \\
P_{y}=-a \omega^{2} \sin \omega t+\frac{\alpha a \sin \omega t}{\left((a \cos \omega t)^{2}+(a \sin \omega t)^{2}+\left(R_{c}+h+w^{*} t\right)^{2}\right)^{(3 / 2)}}, \\
P_{z}=\frac{\alpha\left(R_{c}+h+w^{*} t\right)}{\left((a \cos \omega t)^{2}+(a \sin \omega t)^{2}+\left(R_{c}+h+w^{*} t\right)^{2}\right)^{(3 / 2)}} .
\end{gathered}
$$

Начальные условия запишем в виде

$$
\begin{gathered}
x(0)=x_{0}=a, y(0)=y_{0}=0, z(0)=z_{0}=R_{c}+h, \\
\dot{x}(0)=u_{0}=0, \dot{y}(0)=v_{0}=a \omega, \dot{z}(0)=w_{0}=w^{*},
\end{gathered}
$$

где $h$ - высота атмосферы Земли.

Постановка задачи (1), (2) специально выбрана в виде допускающем точное решение

$$
x^{*}=a \cos \omega t, y^{*}=a \sin \omega t, z^{*}=R_{c}+h+w^{*} t .
$$

Это позволяет вычислить абсолютную погрешность местоположения космического корабля, его скорости и ускорения при применении метода быстрых разложений.

В задаче (1), (2) неизвестными являются координаты центра масс космического корабля $x(t), y(t), z(t)$.

Полагаем, что время изменяется в пределах $t \in\left[0, t_{0}\right]$, где $t_{0}$ - время движения корабля. Для решения нелинейной задачи Коши (1), (2) методом быстрых разложений каждую неизвестную функцию следует представить суммой специальной граничной функции $M_{2 p}$ [3] и ряда Фурье на заданном отрезке $t \in\left[0, t_{0}\right]$, т.е.

$$
\begin{aligned}
& x(t)=x(0)\left(1-\frac{t}{t_{0}}\right)+x\left(t_{0}\right) \frac{t}{t_{0}}+\ddot{x}(0)\left(\frac{t^{2}}{2}-\frac{t^{3}}{6 t_{0}}-\frac{t t_{0}}{3}\right)+\ddot{x}\left(t_{0}\right)\left(\frac{t^{3}}{6 t_{0}}-\frac{t t_{0}}{6}\right)+\sum_{m=1}^{N} x_{m} \sin m \pi \frac{t}{t_{0}}, \\
& y(t)=y(0)\left(1-\frac{t}{t_{0}}\right)+y\left(t_{0}\right) \frac{t}{t_{0}}+\ddot{y}(0)\left(\frac{t^{2}}{2}-\frac{t^{3}}{6 t_{0}}-\frac{t t_{0}}{3}\right)+\ddot{y}\left(t_{0}\right)\left(\frac{t^{3}}{6 t_{0}}-\frac{t t_{0}}{6}\right)+\sum_{m=1}^{N} y_{m} \sin m \pi \frac{t}{t_{0}}, \\
& z(t)=z(0)\left(1-\frac{t}{t_{0}}\right)+z\left(t_{0}\right) \frac{t}{t_{0}}+\ddot{z}(0)\left(\frac{t^{2}}{2}-\frac{t^{3}}{6 t_{0}}-\frac{t t_{0}}{3}\right)+\ddot{z}\left(t_{0}\right)\left(\frac{t^{3}}{6 t_{0}}-\frac{t t_{0}}{6}\right)+\sum_{m=1}^{N} z_{m} \sin m \pi \frac{t}{t_{0}},
\end{aligned}
$$

где $N$-количество членов в рядах Фурье.

В (4) использована граничная функция $M_{2}$, которая увеличивает сходимость ряда Фурье и гарантирует возможность его двукратного дифференцирования по времени.

В результате применения быстрых разложений (4) приходим к задаче о нахождении следующих $12+3 N$ неизвестных констант

$$
\begin{gathered}
x(0), x\left(t_{0}\right), \ddot{x}(0), \ddot{x}\left(t_{0}\right), y(0), y\left(t_{0}\right), \ddot{y}(0), \ddot{y}\left(t_{0}\right), z(0), z\left(t_{0}\right), \ddot{z}(0), \ddot{z}\left(t_{0}\right), \\
x_{m}, y_{m}, z_{m}, \quad m=1 \div N,
\end{gathered}
$$

шесть из которых найдем из граничных условий (2), а остальные $3 N+6$ - из системы нелинейных алгебраических уравнений, получаемой применением оператора быстрых разложений [3] и поточечного метода вычисления коэффициентов быстрых разложений [11-17].

На внеатмосферном участке траектории вычислительные эксперименты проводились при различном количестве $N$ членов ряда Фурье. Траектория полета космического корабля рассчитывалась при $\omega=2 \pi / 7200 c^{-1}, w^{*}=2000 i / \tilde{n}, \quad a=100 i$, 
$g=9,8 \mathrm{M} / \mathrm{c}^{2}, \quad R_{c}=6372$ км, $h=118$ км для различных значений времени полета $t_{0}$. Абсолютные погрешности местоположения корабля, его скорости и ускорения вычислялись соответственно по формулам

$$
\begin{gathered}
\delta s=\sqrt{\left(x^{*}-x\right)^{2}+\left(y^{*}-y\right)^{2}+\left(z^{*}-z\right)^{2}}, \delta v=\sqrt{\left(\dot{x}^{*}-\dot{x}\right)^{2}+\left(\dot{y}^{*}-\dot{y}\right)^{2}+\left(\dot{z}^{*}-\dot{z}\right)^{2}}, \\
\delta a=\sqrt{\left(\ddot{x}^{*}-\ddot{x}\right)^{2}+\left(\ddot{y}^{*}-\ddot{y}\right)^{2}+\left(\ddot{z}^{*}-\ddot{z}\right)^{2}} .
\end{gathered}
$$

Максимальные значения величин $\delta s, \delta v$ и $\delta a$ для внеатмосферного участка траектории приведены в табл. 1 .

Таблица 1

Максимальные значения $\delta s, \delta v$ и $\delta a$

\begin{tabular}{|c|c|c|c|c|}
\hline$t_{0}, \mathrm{c}$ & $N$ & $\delta s_{\max }, \mathrm{M}$ & $\delta v_{\max }, \mathrm{m} / \mathrm{c}$ & $\delta a_{\max }, \mathrm{M} / \mathrm{c}^{2}$ \\
\hline \multirow{3}{*}{14440} & 10 & 6,80 & $2,65 \cdot 10^{-3}$ & $4,26 \cdot 10^{-6}$ \\
\cline { 2 - 5 } & 20 & 0,97 & $3,62 \cdot 10^{-4}$ & $1,08 \cdot 10^{-6}$ \\
\cline { 2 - 5 } & 30 & 0,30 & $1,27 \cdot 10^{-4}$ & $4,84 \cdot 10^{-7}$ \\
\hline \multirow{3}{*}{36000} & 20 & 36,32 & $5,93 \cdot 10^{-3}$ & $7,53 \cdot 10^{-6}$ \\
\cline { 2 - 5 } & 25 & 16,69 & $2,98 \cdot 10^{-3}$ & $4,71 \cdot 10^{-6}$ \\
\cline { 2 - 5 } & 30 & 9,09 & $1,74 \cdot 10^{-3}$ & $3,21 \cdot 10^{-6}$ \\
\hline
\end{tabular}

Видно, что с увеличением числа членов ряда Фурье точность расчетов быстро возрастает. Кроме этого точность расчета можно повысить, выбрав в быстром разложении граничную функцию более высокого порядка [11-17]. Также, следует отметить, что увеличение в расчетах времени полета не приводит к существенному росту численных и временных затрат на ЭВМ.

Таким образом, высокая точность расчетов позволит проводить меньшее количество корректировок траектории и тем самым существенно экономить топливо на космическом корабле, что в свою очередь расширяет возможности космических исследований и принесет большой экономический эффект.

\section{References:}

1. Карагодин В.В. Приближенные методы расчета внеатмосферного активного участка траектории // Электронный журнал «Труды МАИ». Выпуск № 66, 2013. http://www.mai.ru/science/trudy/

2. Хайрер Э., Нёрсетт С., Ваннер Г. Решение обыкновенных дифференциальных уравнений. Нежесткие задачи. М.: Мир. 1990. 512 с.

3. Чернышов А.Д. Метод быстрых разложений для решения нелинейных дифференциальных уравнений // Журнал вычислительной математики и математической физики Т. 54. № 1. 2014. С. 13 - 24.

4. Чернышов А.Д., Марченко А.Н., Горяйнов В.В. Температурный режим при естественной конвекции термовязкой несжимаемой жидкости в емкости прямоугольной формы // Тепловые процессы в технике, 2012 г., Т. 4, №11, С. 482-486.

5. Чернышов А.Д., Павлов И.О., Воронова Е.В., Горяйнов В.В. Решение методом быстрых разложений задачи о сушке зерна // Теплофизика и аэромеханика, 2012, том 19, № 6. C. 739-749. 
6. Чернышов А.Д., Горяйнов В.В. Решение одного нелинейного интегродифференциального уравнения методом быстрых разложений // Вестник ЧГПУ им. И.Я. Яковлева. Серия: механика предельного состояния. № 4(12). 2012. С. 105 - 112.

7. Чернышов А.Д., Попов В.М., Шахов А.С., Горяйнов В.В., Новиков А.П. Решение задачи о контактном тепловом сопротивлении между сжатыми шарами методом быстрых разложений // Тепловые процессы в технике Т. 4, № 12. 2012. С. 544 - 552.

8. Чернышов А.Д., Марченко А.Н., Горяйнов В.В. Решение задачи о деформировании термоупругой пластины методом быстрых разложений// Вестник Воронежского государственного университета инженерных технологий. 2013. № 2. С. 84-89.

9. Попов В.М., Шахов А.С., Горяйнов В.В., Чернышов О.А., Новиков А.П. Повышенная точность решения задачи о контактном термосопротивлении между сжатыми шарами методом быстрых разложений // Тепловые процессы в технике -Т. 6 - № 4. - 2014. C. $179-191$.

10. Чернышов А.Д., Горяйнов В.В. Чернышов О.А. Решение задачи о полете космического корабля в атмосфере Земли методом быстрых разложений // Тенденции развития технических наук: сборник статей Международной научно- практической конференции - Уфа: Аэтерна, 2014. -С. 82 - 85.

11. Чернышов А.Д., Горяйнов В.В., Соловьев А.О. О возможности вычисления коэффициентов Фурье поточечным методом.// Вестник Воронежского государственного технического университета. Т. 6. № 2. 2010. С. 49 - 53.

12. Горяйнов В.В. Устойчивость поточечного метода вычисления коэффициентов быстрых рядов Фурье // Актуальные проблемы прикладной математики, информатики и механики / Сб. тр. междунар. конф. - Воронеж.: ВГУ, 2010 г. С. 120 - 124.

13. Горяйнов В.В. Анализ погрешности быстрых рядов Фурье при их многократном дифференцировании для случая вычисления коэффициентов ряда поточечным методом // Вестник Воронежского государственного технического университета. Т. 7. № 2. 2011. С. $36-40$.

14. Чернышов А.Д., Хозяинова Н.А., Горяйнов В.В. Исследование погрешности поточечного метода вычисления коэффициентов быстрых рядов Фурье // Вестник Воронежской государственной технологической академии. Сер. Информационные технологии, моделирование и управление. № 2. 2011. С. 64 - 67.

15. Чернышов А.Д., Горяйнов В.В. О выборе оптимального порядка граничной функции в быстром разложении // Вестник Воронежского государственного университета. Серия: Системный анализ и информационные технологии. 2011. №1. С. 60 - 65 .

16. Чернышов А.Д., Горяйнов В.В. О сравнении быстрых синус - и косинус-разложений в краевых задачах с условиями Дирихле // Актуальные проблемы прикладной математики, информатики и механики / Сб. тр. междунар. конф. - Воронеж.: ВГУ, 2011 г. С. $417-422$.

17. Чернышов А.Д., Горяйнов В.В. О способе нанесения расчетных точек на отрезок при реализации поточечного метода вычисления коэффициентов быстрых разложений для решения краевой задачи с условиями Дирихле // Вестник Воронежского государственного университета. Серия: Системный анализ и информационные технологии. № 2. 2012. С $30-35$. 